Triclinic layered $\mathrm{A}_{2} \mathrm{ZnSi}_{3} \mathrm{~S}_{8}(\mathrm{~A}=\mathrm{Rb}, \mathrm{Cs})$ with large optical anisotropy and systematic research on inherent structure-performance relationship in $\mathrm{A}_{2} \mathrm{M}^{\mathrm{IIB}} \mathrm{M}^{\mathrm{IV}}{ }_{3} \mathrm{Q}_{8}$ family

Lihua Gao, Ya Yang, Bingbing Zhang, ${ }^{*}$ Xiaowen $\mathrm{Wu},{ }^{*} \mathrm{Kui} \mathrm{Wu}$

Key Laboratory of Medicinal Chemistry and Molecular Diagnosis of the Ministry of Education, Key Laboratory of Analytical Science and Technology of Hebei Province, College of Chemistry and Environmental Science, Hebei University, Baoding 071002, China.

E-mail: wukui@hbu.edu.cn (Kui Wu); zhangbb@hbu.edu.cn (Bingbing Zhang); wuxiaowen1114@163.com (Xiaowen Wu).

\title{
CONTENTS
}

1. Synthesis of Title Compounds

2. Structural Refinement and Crystal Data

3. Property Characterization 


\section{Synthesis of Title Compounds}

Raw materials including the $\mathrm{CsCl}$ (99\%), $\mathrm{RbCl}$ (99.9\%), $\mathrm{ZnS}$ (99.99\%), $\mathrm{SiS}_{2}(99.99 \%)$ and S powder (99.99\%) were used as purchased from Beijing Hawk Science \& Technology Co., Ltd. Raw mixtures with $1 \mathrm{~mol} \mathrm{ZnS,} 3$ mol SiS 2,1 mol S, and excess $3 \mathrm{~mol} \mathrm{CsCl}$ or $\mathrm{RbCl}$ were loaded into the graphite crucibles and then put them into vacuum-sealed silica tubes. Muffle furnace was used to complete the spontaneous crystallization and detailed setting temperature process was shown as following: firstly heated up to $750{ }^{\circ} \mathrm{C}$ in $40 \mathrm{~h}$ to ensure the mixture melt completely and kept at this temperature within 5 days, then slowly cooled down to room temperature in 6 days. Finally, many submillimeter-level colorless crystals were found under the optical microscope after the wash with $\mathrm{N}, \mathrm{N}$-dimethylformamide (DMF) solution. Deionized water was also used to remove the excess $\mathrm{CsCl}$ or $\mathrm{RbCl}$. Both of them are stable in the air within several months.

\section{Structural Refinement and Crystal Data}

Selected high-quality crystals were used for data collections on a Bruker SMART APEX II 4K CCD diffractometer using Mo K $\alpha$ radiation $(\lambda=0.71073 \AA)$ at $296 \mathrm{~K}$. The crystal structures were solved by direct method and refined using the SHELXTL program package. Multi-scan method was used for absorption correction. PLATON was also used to check the final structures and no other symmetries were found. Detail refinement parameters and data were shown in Table 1.

\section{Property Characterization}

\subsection{Powder X-ray Diffraction}

Powder X-ray diffraction (XRD) patterns of title compounds were collected on a Bruker D2 X-ray diffractometer with $\mathrm{Cu} \mathrm{K \alpha}$ radiation $(\lambda=1.5418 \AA)$ at room temperature. The $2 \theta$ range was $10-70^{\circ}$ with a step size of $0.02^{\circ}$ and a fixed counting time of $1 \mathrm{~s} /$ step.

\subsection{UV-Vis-Near-IR (NIR) Diffuse-Reflectance}

Diffuse-reflectance spectra were measured by a Shimadzu SolidSpec-3700DUV spectrophotometer in the wavelength range of 200-2600 $\mathrm{nm}$ at room temperature.

\subsection{Thermal analysis}


A HCT-2 (HENVEN) thermal analyzer was used to investigate their differential scanning calorimetric (DSC) curves. About $5 \mathrm{mg}$ microcrystals were placed into a vacuum-sealed silica tube $\left(\Phi 4 \times 2 \mathrm{~mm}^{3}\right)$. The measurement ranges are located at the temperature from 25 to 700 or $600{ }^{\circ} \mathrm{C}$. The temperature rates in heating and cooling process were $7^{\circ} \mathrm{C} / \mathrm{min}$, respectively.

\subsection{Computational Description}

In order to further investigate the relationship of structure-property, their electronic structures were studied by density functional theory (DFT). The exchange-correlation potential was calculated by Perdew-Burke-Ernzerhof (PBE) functional within the generalized gradient approximation (GGA) with the scheme. To achieve energy convergence, a plane-wave basis set energy cutoff was $900 \mathrm{eV}$ within normal-conserving pseudo-potential (NCP). As important parameters for IR crystals, SHG coefficient and birefringence were calculated with suitable scissors operators. 
Table S1 Coordination parameters of $\mathrm{AS}_{n}$ and $d(\mathrm{~A}-\mathrm{S})$ in series of compounds.

\begin{tabular}{llll}
\hline & Space group & $\mathrm{AS}_{n}$ & $d(\mathrm{~A}-\mathrm{S})(\AA)$ \\
\hline $\mathrm{Cs}_{2} \mathrm{ZnSi}_{3} \mathrm{~S}_{8}$ & $P-1$ & $n=9,10$ & $3.412-4.048$ \\
$\mathrm{~K}_{2} \mathrm{ZnGe}_{3} \mathrm{~S}_{8}$ & $P 2_{1}$ & $n=7,8$ & $3.127-3.721$ \\
$\mathrm{Cs}_{2} \mathrm{ZnGe}_{3} \mathrm{~S}_{8}$ & $P 2_{1} / n$ & $n=9$ & $3.438-4.060$ \\
$\mathrm{Cs}_{2} \mathrm{CdGe}_{3} \mathrm{~S}_{8}$ & $P 2_{1} 2_{1} 2_{1}$ & $n=10$ & $3.463-4.114$ \\
\hline
\end{tabular}

Table S2 Melting and crystallization temperatures in 2-1-3-8 series of compounds.

\begin{tabular}{lll}
\hline & Melting temperature $\left({ }^{\circ} \mathrm{C}\right)$ & Crystallization temperature $\left({ }^{\circ} \mathrm{C}\right)$ \\
\hline $\mathrm{Cs}_{2} \mathrm{HgSn}_{3} \mathrm{Se}_{8}$ & 595 & 577 \\
$\mathrm{~K}_{2} \mathrm{ZnGe}_{3} \mathrm{~S}_{8}$ & 750 & 690 \\
$\alpha-\mathrm{K}_{2} \mathrm{ZnSn}_{3} \mathrm{~S}_{8}$ & 468 & 398 \\
$\mathrm{Cs}_{2} \mathrm{ZnSi}_{3} \mathrm{~S}_{8}$ & 521 & 504 \\
$\mathrm{Rb}_{2} \mathrm{ZnSi}_{3} \mathrm{~S}_{8}$ & 631 & 610 \\
\hline
\end{tabular}

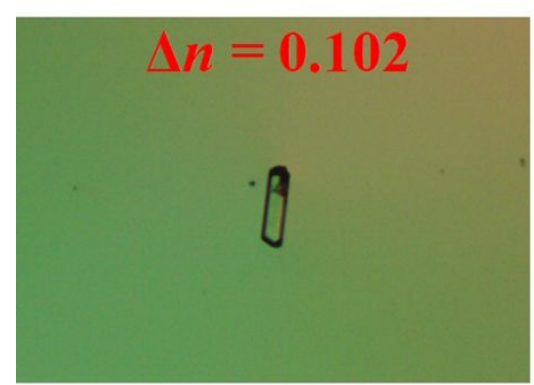

$\mathbf{R b}_{2} \mathrm{ZnSi}_{3} \mathrm{~S}_{8}$

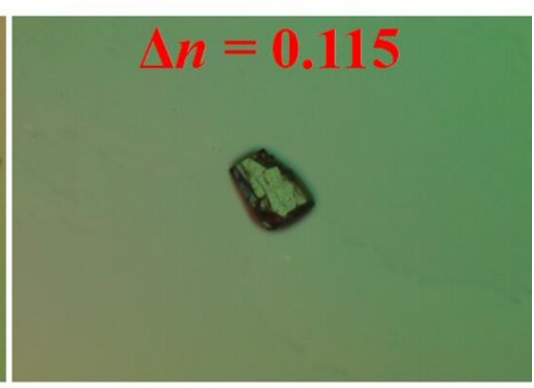

$\mathrm{Cs}_{2} \mathrm{ZnSi}_{3} \mathrm{~S}_{8}$

Figure $\mathbf{S 1}$ Birefringence determination for $\mathrm{Rb}_{2} \mathrm{ZnSi}_{3} \mathrm{~S}_{8}$ and $\mathrm{Cs}_{2} \mathrm{ZnSi}_{3} \mathrm{~S}_{8}$. 